\title{
Neural Network Based Approach for the Generation of Road Feel in a Steer-By-Wire System
}

\author{
Jayachandran $^{\mathrm{a}}$ and Denis Ashok ${ }^{\mathrm{b}, *}$ \\ School of Mechanical Engineering, VIT University, Vellore, Tamil Nadu 632 014, India \\ E-mail: achandrugtec@gmail.com, bdenisashok@vit.ac.in (Corresponding author)
}

\begin{abstract}
Steer-by-wire is an advanced steering system which connects the steering wheel with the front wheel by using motors and sensors. Generating a variable road feel to the driver in a steer by wire system is an important task as there is no mechanical connection; it requires the development of suitable feedback algorithm for producing variable torque feedback in the steering wheel. In present work, Neural Network method is proposed for estimating the current to be supplied to the feedback motor using the vehicle dynamic parameters such as vertical displacement of front axle, self-aligning moment and front wheel angle as input parameters. A simulation study has been carried out for verifying the performance of the proposed method using the vehicle dynamic models available in the literature. It was tested for dry, wet and icy road profiles using the typical model parameters. The simulated vehicle dynamic parameters and estimated motor current values for the different road surfaces using neural network are presented. From the simulation results for the sinusoidal road surface and sinusoidal steering angle driver input, it is clear that the neural network based method is able to produce the varying road feel to the driver for the different road conditions.
\end{abstract}

Keywords: Steer-by-wire, vehicle dynamics, neural network, road feel, feedback.

ENGINEERING JOURNAL Volume 20 Issue 5

Received 30 October 2015

Accepted 28 January 2016

Published 25 November 2016

Online at http://www.engj.org/

DOI:10.4186/ej.2016.20.5.49 


\section{Introduction}

Steering system is an important element in a vehicle which connects the vehicle with the road. In order to improve the efficiency of the steering systems, many improvements have been done over the time. In many such improvements, steer-by-wire is known as one of the advanced steering system technique which does not have any mechanical link between the steering wheel and the front wheel. It achieves the vehicle maneuvering using motors and sensors. Safety and reduction in cost are advantages of this advanced steering technique. However, producing the steering feel to the driver is the main concern of steer by wire vehicles due to removal of mechanical link between the steering wheel and road wheel. Generation of the road feel requires development of feedback algorithms to produce the road feel to the driver.

Various algorithms and techniques have been proposed by many researchers for producing the road feel to the driver in steer by wire systems. Shengbing et al. proposed a steering ratio control method which uses the vehicle dynamic behaviour for producing the steering feel using the steering angle, reactive torque of road wheels [1]. Eid and Saeed proposed a combined system of power steering and a steer by wire system based on the active steering angle control. They designed an optimal controller to monitor the dynamic behaviour of the steer by wire. Linear quadratic regulator and PID were used [2]. Wang et al. proposed a new feedback control with the consideration of real time lateral acceleration and yaw rate. The strategy of variable gear ratio was also used and the double lane experiment was done using computer simulation technique [3]. Hongyu et al. developed a new feedback control for producing the steering feel using active damping control technique and assisting characteristic method for an electronic power steering system [4]. Reza and Iman developed a new modelling technique for steer-by-wire system using the bond graph theory [5]. Bing and Sohel proposed a fault tolerant method for steer by wire system which neglects the single point errors and does not affect the road-wheel control performance of the system. Dual motors and microcontrollers were used which reduces the power into half and the complexity of the system [6]. Iman and Reza controlled the yaw rate of steer by wire system by designing two different controllers. The authors proposed a PID controller for the steering wheel and sliding mode controller for the front wheels. The complete steer by wire system was modeled using bond graph method [7].Yandong et al. proposed a method for controlling the steering wheel torque by torque ratio. This method converts the torque ratio into assisting torque by analyzing the characteristic relationship. The authors verified the proposed method for various working conditions which proved the steering wheel torque obtained was similar to the expected result [8]. Hsiaohsiang et al. designed a cornering algorithm for improving the performance and the maneuvering of steer by wire system. Evaluations were done using driving simulators and the results were compared with the conventional steering system. Optimization approach was also produced for road feel by using modified steering effort [9]. Marouf et al. developed a sliding mode controller for electric power steering with the unknown inputs to estimate the driver torque and the reaction force of road. A brushed motor was used for the controller [10]. Sheikh et al. estimated the torque at the steering wheel and the front wheel by using the direct current measurement technique. They designed a linear quadratic regulator to control the feedback for producing realistic feedback torque to the driver [11]. Peng et al. analyzed the tire-road interaction with the help of various tire lateral acceleration dynamic models such as string model, beam model and spring-damper model [12]. Andronic et al. used the quarter car model for analyzing the effect of road inputs for a passive suspension. The ride and handling characteristics were also tested [13]. Bajcinca et al. modeled a friction forces by using the stochastic Gauss-Markov process and also with the kalman filter technique. The non-linear model was used for the simulation process for the two input conditions such as rectangular torque waveform and rectangular torque with the disturbance [14]. It was understood that mathematical models based on vehicle dynamics were developed for steer by wire vehicles. However, these models require basic assumptions and estimation of model parameters for understanding the system behaviour.

In order to produce the realistic steering feel to the driver in steer by wire vehicles, artificial intelligence techniques were developed for steer-by-wire systems. Paolo et al. proposed a method of simulating tire and suspension systems by using recurrent neural networks to analyze the vibration and harshness parameters for different suspension operating conditions [15]. Ikbal and Ali developed a neural network based robust control system for an active steering system. In order to improve the safety, four different control structures were used such as model based neural controller, predictive controller, robust predictive control system and PID controller [16]. Junaid et al. proposed a cosine function neural network method by taking tire-road signal as an error to the system [17]. Na et al. developed a neural network based system for the identification of driver behaviour characteristics [18]. Meng et al. proposed fault diagnosis model for steer- 
by-wire system by training the neural network and compared with the genetic algorithm [19]. Jeong et al. developed a hybrid neural network model for vehicle dynamic simulation of a bushing suspension model [20]. Lin et al. proposed a neural network model for driver handling behaviour and conducted study on the interaction between the driver and the environment [21]. Judycki and Jaskula discussed the mechanistic and empirical methods for analysing the effect of load transfer of vehicle on different road surfaces and pavements [22].

It can be seen that many research works have been focused on developing a road feedback control system for steer-by-wire system by considering various vehicle dynamic parameters such as yaw rate, lateral acceleration and vehicle velocity. However, these methods are not able to generate the road information to the driver because these approaches do not include the tire and road conditions. In order to overcome all these issues, this paper develops a neural network based model which includes the vertical displacement due to the road disturbances, the front wheel angle and the aligning moment as inputs. The feedback motor current is estimated as an output from the proposed neural network controller for the different road conditions. To analyze the performance of the proposed method, a simulation study has been done using vehicle dynamic models for various road conditions.

\section{Modelling of Steer-by-Wire System}

As the road feel is the response proportionality to the variations in the dynamic states of the vehicle, this section provides vehicle dynamic modelling of a typical steer-by-wire system for analyzing the lateral and vertical dynamics of the vehicle. The major elements of a steer by wire system consists of steering wheel, feedback motor, electronic control unit, front wheel motor, axles, rack and pinion arrangement, front wheel as shown in Fig. 1.

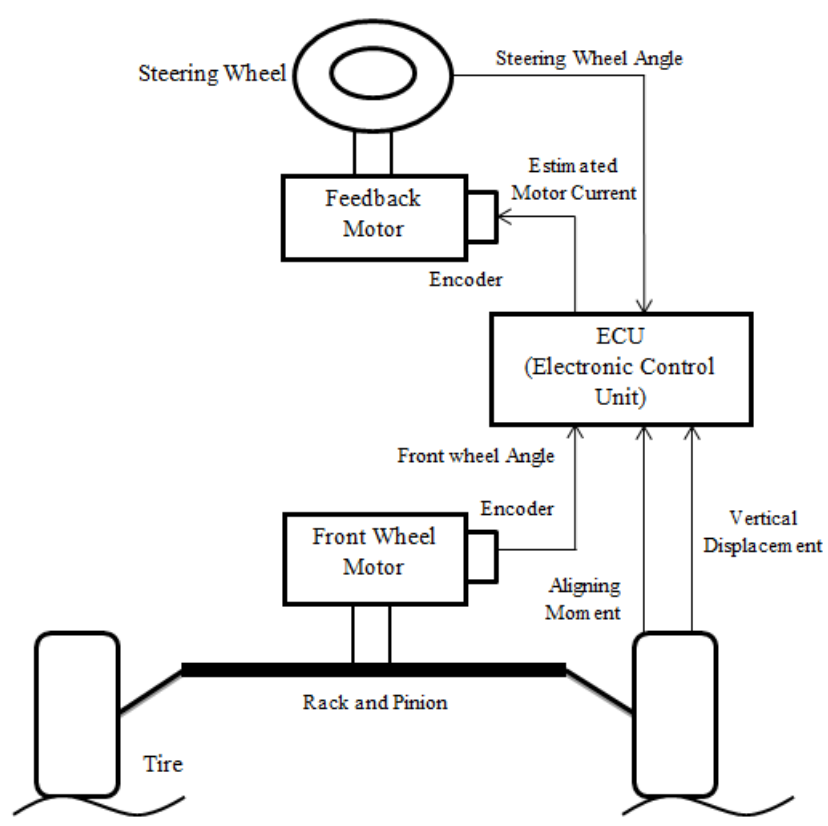

Fig. 1. Structure of the steer-by-wire system [11].

In order to generate the road feel to the driver, a feedback motor is used to provide an artificial torque feedback in the steering wheel. Electronic control unit needs to provide the required current to the motor for producing the torque feedback to the driver based on the changing road conditions. In the present work, vehicle dynamic parameters such as front wheel angle and vertical displacement of front axle along with aligning moment of the vehicle are considered for producing the variable road feel to the driver. 


\subsection{Front Wheel Angle of the Vehicle}

Front wheel angle is an important vehicle parameter which defines the lateral dynamic state of the vehicle for the given driver input. In order to produce the better road feel to the driver, front wheel angle has been used as feedback parameter in steer by wire vehicles [1]. The front wheel sub system includes a front wheel and front wheel motor [2] which is shown in Fig. 2. The dynamic model of front wheel is given as in Eq. (1) and simplified as in Eq. (2),

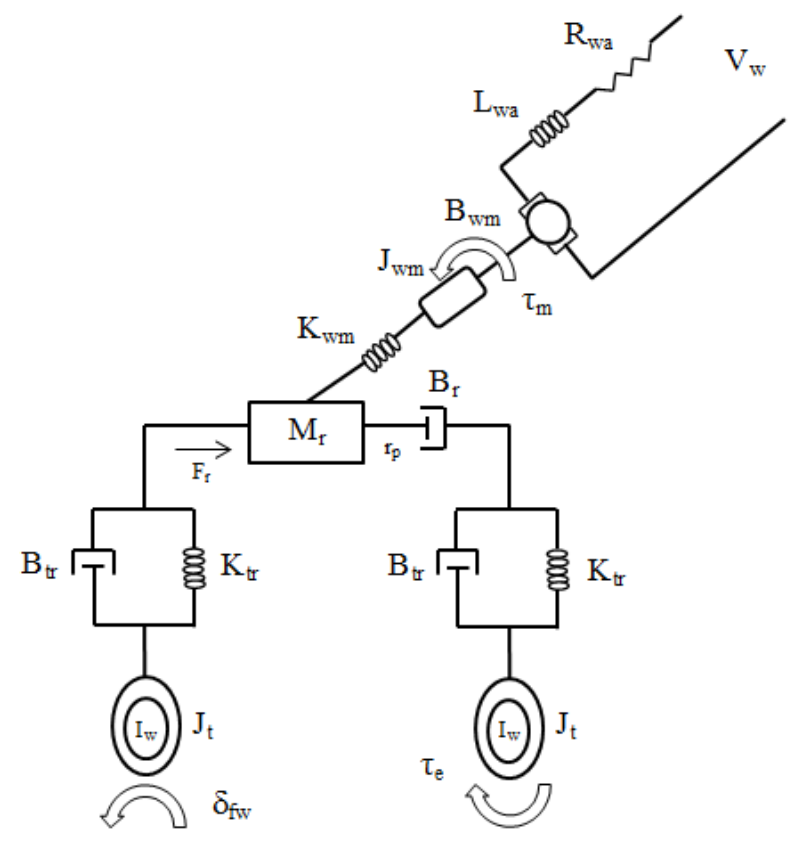

Fig. 2. Physical model of the front wheel system [5].

The differential equation of front wheel model is given as in Eq. (1) and Eq. (2),

$$
J_{w m} \ddot{\delta}_{f w}+B_{w m} \dot{\delta}_{f w}+F_{r} r_{p}+\tau_{e}=\tau_{m}
$$

By simplifying,

$$
J_{w m} \ddot{\delta}_{f w}+B_{w m} \dot{\delta}_{f w}=\tau_{m}-\tau_{e}-F_{r} r_{p}
$$

where $J_{w m}$ is the inertia of road wheel system, $\delta_{\mathrm{fw}}$ is the front wheel angle, $\mathrm{B}_{\mathrm{wm}}$ is the viscous friction coefficient, $F_{\mathrm{r}}$ is rack force, $\tau_{\mathrm{e}}$ is the self-aligning moment, $\mathrm{r}_{\mathrm{p}}$ is radius of pinion and $\tau_{\mathrm{m}}$ is the motor torque.

The dynamic equations are represented as a second order derivative which makes the system modelling difficult. Since the front wheel system consists of both electrical and mechanical elements, the modelling can be done by using the bond graph approach. It divides the system into many subsystems and shows the each component's energetic interconnection. The bond graph modelling of a front wheel system is shown in Fig. 3, where TF is known as a transformer which conserves the power, $\mathrm{p}$ is known as the potential variables and $\mathrm{q}$ is represented as flow variable. 


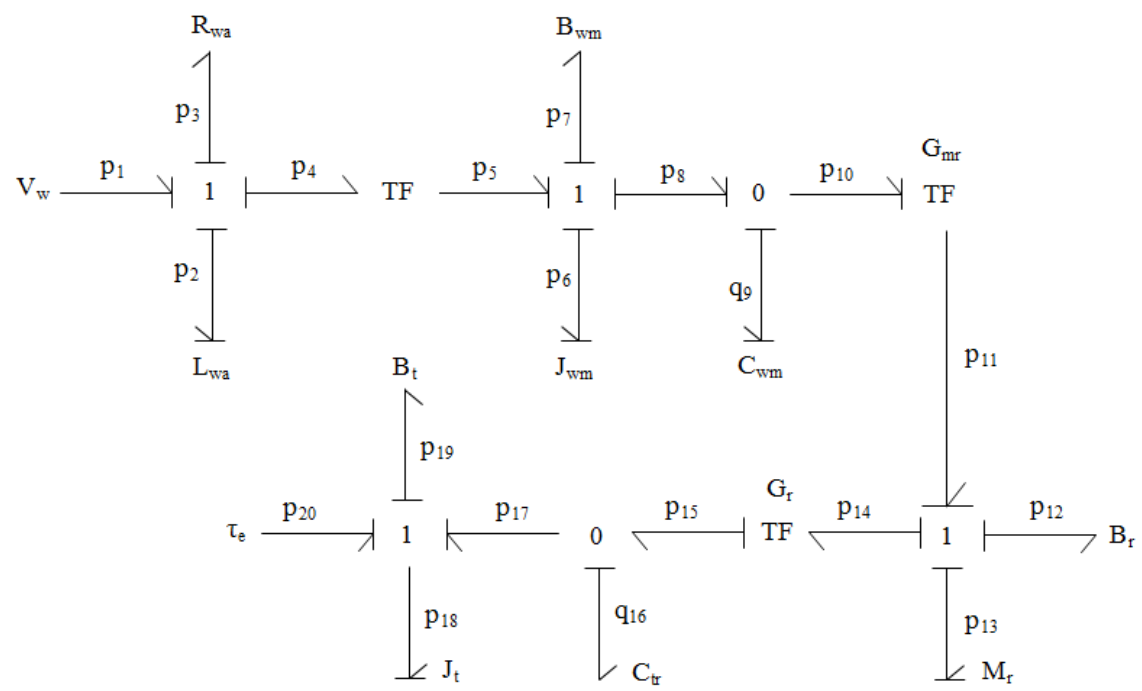

Fig. 3. Bond graph model for the front wheel system [5].

The state equations of the front wheel model using the bond graph method [5] are explained in the following section. The voltage of the front wheel motor is given as in Eq. (3),

$$
\dot{p}_{2}=-\frac{R_{w a}}{L_{w a}} p_{2}-\frac{K_{w m}}{J_{w m}} p_{6}+V_{w}
$$

The front wheel motor torque and the angular velocity of the front wheel motor are mentioned as in Eq. (4) and Eq. (5),

$$
\begin{gathered}
\dot{p}_{6}=\frac{K_{w m}}{L_{w a}} p_{2}-\frac{B_{w m}}{J_{w m}} p_{6}-\frac{1}{C_{w m}} q_{9} \\
\dot{q}_{9}=\frac{1}{J_{w m}} p_{6}-\frac{1}{G_{m r} M_{r}} p_{13}
\end{gathered}
$$

The rack force and linear velocity of the tie-rod are calculated as in Eq. (6) and Eq. (7),

$$
\begin{gathered}
\dot{p}_{13}=-\frac{B_{r}}{M_{r}} p_{13}+\frac{1}{G_{m r} C_{w m}} q_{9}-\frac{G_{r}}{C_{t r}} q_{16} \\
\dot{q}_{16}=\frac{1}{G_{r} M_{r}} p_{13}-\frac{1}{J_{t}} p_{18}
\end{gathered}
$$

The torque acting on the tire and the front wheel angle are expressed as in Eq. (8) and Eq. (9),

$$
\begin{gathered}
\dot{p}_{18}=-\frac{B_{t}}{J_{t}} p_{18}+\frac{1}{C_{t r}} q_{16} \\
\delta_{f w}=\frac{1}{J_{t}} p_{18}
\end{gathered}
$$

where $p_{2}$ is Front wheel motor voltage, $p_{6}$ is Torque of front wheel motor, $q_{9}$ is Angular velocity of front wheel motor, $p_{13}$ is Rack force, $q_{16}$ is Linear velocity of the tie-rod, $p_{18}$ is Torque acting on the tire, $\delta_{f w}$ is Front wheel angle. The bond graph variables can be represented as a state space form [23] which is given as in Eq. (10) and Eq. (11),

$$
\begin{aligned}
& \dot{X}_{w}(t)=A X_{w}(t)+B u_{w}(t) \\
& y_{w}(t)=C X_{w}(t)+D u_{w}(t)
\end{aligned}
$$

By combining the state equations, the state vector is represented as in Eq. (12),

$$
\mathrm{X}_{\mathrm{w}}=\left[\begin{array}{llllll}
p_{2} & p_{6} & q_{9} & p_{13} & q_{16} & p_{18}
\end{array}\right]
$$


The voltage for the front wheel motor and the torque produced by the road are considered are the inputs to the state space model which is given as in Eq. (13), the front wheel angle is considered as an output of the model is given as in Eq. (14),

$$
\begin{gathered}
\mathrm{u}_{\mathrm{w}}=\left[\begin{array}{ll}
V_{w} & \tau_{e}
\end{array}\right] \\
\mathrm{A}=\left[\begin{array}{cccccc}
\frac{-R_{w a}}{L_{w a}} & \frac{-K_{w m}}{K_{w m}} & 0 & 0 & 0 & 0 \\
\frac{K_{w m}}{L_{w a}} & \frac{-B_{w m}}{J_{w m}} & 0 & 0 & \frac{-1}{C_{w m}} & 0 \\
0 & 0 & \frac{-B_{r}}{M_{r}} & 0 & \frac{1}{G_{m r} C_{w m}} & \frac{-G_{r}}{C_{t r}} \\
0 & 0 & 0 & \frac{-B_{t r}}{I_{w}} & 0 & \frac{1}{C_{t r}} \\
0 & \frac{1}{J_{w m}} & \frac{-1}{G_{m r} M_{r}} & 0 & 0 & 0 \\
0 & \frac{1}{G_{r} M_{r}} & \frac{-1}{I_{w}} & 0 & 0
\end{array}\right] \\
\mathrm{B}=\left[\begin{array}{cccccc}
1 & 0 & 0 & 0 & 0 & 0 \\
0 & 0 & 0 & 1 & 0 & 0
\end{array}\right]^{T} \\
\mathrm{C}=\left[\begin{array}{cccccc}
0 & 0 & 0 & 0 & \frac{1}{J_{t}}
\end{array}\right]
\end{gathered}
$$

In the above state space equation $R_{w a}$ is motor resistance, $M_{r}$ is mass of the rack, $G_{m r}$ is radius of the pinion, $G_{r}$ is length ratio of the steering arm, $I_{w}$ is wheel moment of inertia about the spin axis, $B_{t r}$ is resistance of the tie rod, $B_{r}$ is the resistance of the rack, $C_{w m}$ is the compliance of the front wheel motor shaft, $\mathrm{K}_{\mathrm{wm}}$ is the front wheel motor constant, $\mathrm{L}_{\mathrm{wa}}$ is the motor inductance, $\mathrm{C}_{\mathrm{tr}}$ is the compliance of the tie rod. The state space model will be used in calculating the commanded front wheel angle of the vehicle for the given front wheel motor voltage and self-aligning moment.

\subsection{Self-Aligning Moment of Vehicle}

When the front wheels are steered in the road, a reaction torque is developed due to the self-aligning moment and it is experiences as the steering feel by the driver. Since the self-aligning moment has the significant effect on the handling quality and safe driving, it is recognized as an essential feedback to the driver [4]. In the present work, the two degrees of freedom bicycle model is considered for calculating the self-aligning moment of the vehicle [7]. The bicycle model of the vehicle is shown in Fig. 4. 


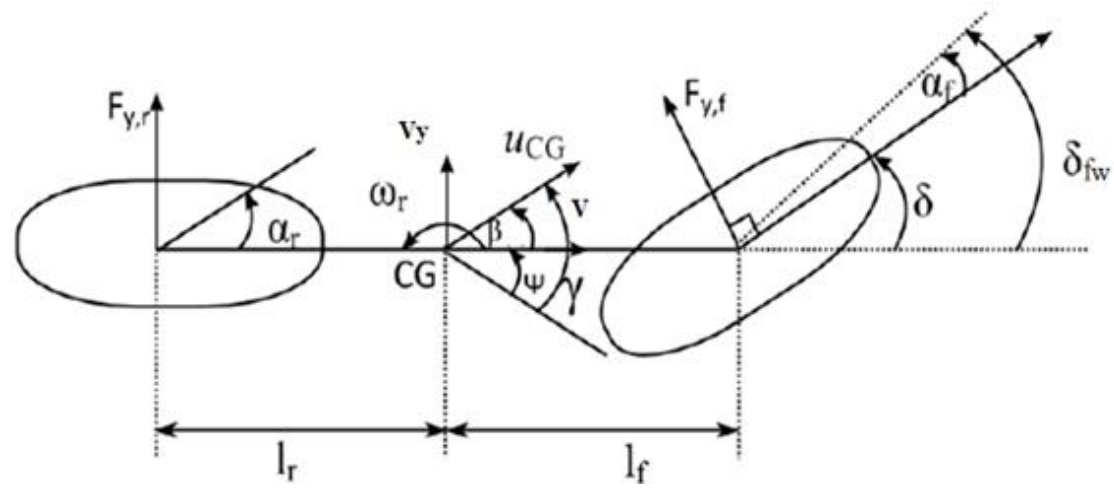

Fig. 4. Bicycle model of the vehicle.

The angle between the center line of the front wheel and the direction of the velocity of the front wheel is defined as the front slip angle, which is denoted as $\alpha_{\mathrm{f}}$. The side slip angle $\beta$ is defined as the angle between the direction of the vehicle velocity and the centre line of the vehicle. $\delta$ is denoted as the steering wheel angle. The lateral force at the front and rear tires are related to the slip angle and cornering stiffness of the tire as Eq. (15) and Eq. (16) respectively.

$$
\begin{aligned}
F_{y, f} & =-C_{\alpha, f} * \alpha_{f} \\
F_{y, r} & =-C_{\alpha, r} * \alpha_{r}
\end{aligned}
$$

The slip angle at the front and rear tires is given as in Eq. (17) and Eq. (18),

$$
\begin{gathered}
\alpha_{f}=\beta+\frac{l_{f} \psi}{V}-\delta_{f w} \\
\alpha_{r}=\beta-\frac{l_{r} \psi}{V}
\end{gathered}
$$

From the above equations, it can be noticed that the slip angle of the vehicle is very much related to the yaw rate $\psi$ and side slip angle $\beta$. Based on the Newton's law of motions, the vehicle model equations of a bicycle model is given as in Eq. (19) and Eq. (20),

$$
\begin{gathered}
m V \dot{\beta}=F_{y, f}+F_{y, r} \\
I_{z} \dot{\psi}=l_{f} F_{y, f}-l_{r} F_{y, r}
\end{gathered}
$$
(19),

Substituting the values of the lateral forces and slip angle values using Eq. (15), (16), (17), (18) in Eq.

$$
m V \dot{\beta}=-C_{\alpha, f}\left(\beta+\frac{l_{f} \psi}{V}-\delta_{f w}\right)-C_{\alpha, r}\left(\beta-\frac{l_{r} \psi}{V}\right)
$$

Simplifying the above equation gives the side slip angle as in Eq. (22),

$$
\dot{\beta}=\left(\frac{-C_{\alpha, f}-C_{\alpha, r}}{m V}\right) \beta+\left(\frac{-C_{\alpha, f} l_{f}+C_{\alpha, r} l_{r}}{m V^{2}}\right) \psi+\left(\frac{C_{\alpha, f}}{m V}\right) \delta_{f w}
$$

Similarly, the yaw rate of the vehicle can be calculated by substituting the values of the lateral forces and slip angle values using Equations (15), (16), (17), (18) in Eq. (23), 


$$
I_{z} \dot{\psi}=l_{f}\left(-C_{\alpha, f}\left(\beta+\frac{l_{f} \psi}{V}-\delta_{f w}\right)\right)+l_{r}\left(C_{\alpha, r}\left(\beta-\frac{l_{r} \psi}{V}\right)\right)
$$

Simplifying the above equation gives the yaw dynamics which is given as in Eq. (24),

$$
\dot{\psi}=\left(\frac{-l_{f} C_{\alpha, f}+l_{r} C_{\alpha, r}}{I_{z}}\right) \beta+\left(\frac{-C_{\alpha, f} l_{f}^{2}-C_{\alpha, r} l_{r}^{2}}{V I_{z}}\right) \psi+\left(\frac{C_{\alpha, f} l_{f}}{I_{z}}\right) \delta_{f w}
$$

Taking the side slip angle $\beta$ and the yaw rate $\psi$ as the states of the system which is shown in Eq. (25) and Eq. (26), the given vehicle model can be derived as in Eq. (29),

where

$$
\begin{aligned}
& \dot{X}_{B}(t)=A X_{B}(t)+B u_{B}(t) \\
& y_{B}(t)=C X_{B}(t)+D u_{B}(t)
\end{aligned}
$$

In the above equation $l_{\mathrm{f}}$ and $l_{\mathrm{r}}$ are distance between the center of gravity to the front wheel and the rear wheel respectively, $\mathrm{I}_{\mathrm{z}}$ is the moment of inertia, $\mathrm{C}_{\alpha, \mathrm{f}}$ and $\mathrm{C}_{\alpha, \mathrm{r}}$ are the cornering stiffness of each front and rear wheels and $\mathrm{V}$ is the vehicle speed. The self-aligning moment can be calculated using the mechanical trail and pneumatic trail which is shown in Eq. (30),

$$
\tau_{e}=F_{y, f}\left(t_{p}+t_{m}\right)
$$

Combining the lateral force and slip angle in Eq. (30) which gives the equation to measure the selfaligning moment is given as in Eq. (31),

$$
\tau_{e}=-C_{f}\left(t_{p}+t_{m}\right)\left(\beta+\frac{\Psi l_{f}}{V}-\delta_{f w}\right)
$$

where $t_{p}$ is pneumatic trail, $t_{m}$ is mechanical trail and $C_{f}$ is the cornering stiffness.

\subsection{Vertical Displacement of the Front Axle}

Road input is an essential part of a steer-by-wire system which gives the road conditions as a feedback to the driver. In order to study the vertical motion of a vehicle for the given road input, two degrees of freedom quarter car model is considered in the present work [8] which is shown in Fig. 5. 


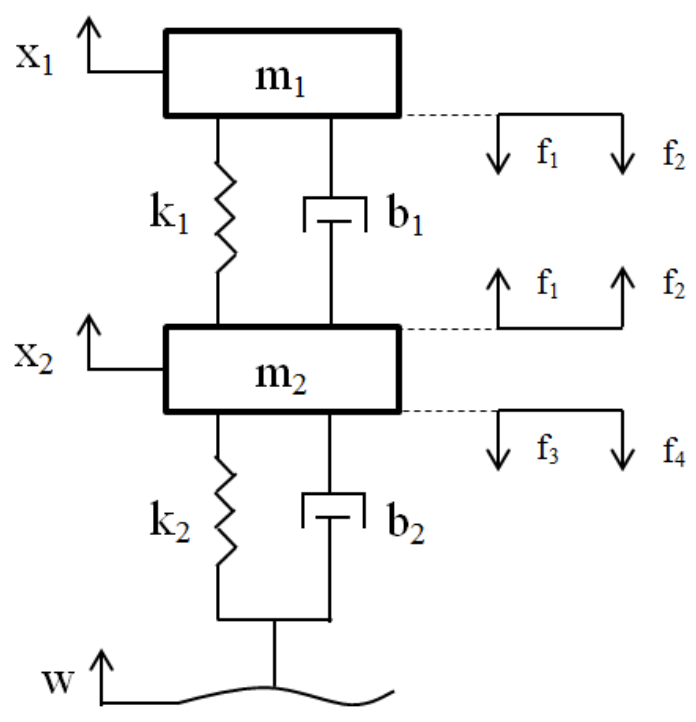

Fig. 5. Quarter car model.

In the above diagram, $\mathrm{m}_{1}$ is the sprung mass, $\mathrm{m}_{2}$ is the unsprung mass, $\mathrm{k}_{1}$ is spring stiffness, $\mathrm{k}_{2}$ is tire stiffness, $b_{1}$ is the damping coefficient of the suspension, $b_{2}$ is the damping coefficient of the tire, $x_{1}$ is the vertical displacement of sprung mass, $\mathrm{x}_{2}$ is the vertical displacement of unsprung mass and $\mathrm{w}$ is the road input.

The vertical displacement of the front axle is calculated using the following equations of motions for the sprung and unsprung mass of the vehicle as given below:

$$
\begin{aligned}
& f_{1}=k_{1}\left(x_{1}-x_{2}\right) \\
& f_{2}=b_{1}\left(\dot{x}_{1}-\dot{x}_{2}\right) \\
& f_{3}=k_{2}\left(x_{2}-w\right) \\
& f_{4}=b_{2}\left(\dot{x}_{2}-\dot{w}\right)
\end{aligned}
$$

Based on the differential equations of motion for sprung mass $\mathrm{m}_{1}$, Eq. (36) is obtained as given below:

$$
\begin{gathered}
m_{1} \ddot{x}_{1}=-f_{1}-f_{2} \\
m_{1} \ddot{x}_{1}=-k\left(x_{1}-x_{2}\right)-b_{1}\left(\dot{x}_{1}-\dot{x}_{2}\right) \\
\ddot{x}_{1}=\frac{1}{m_{1}}\left(-k\left(x_{1}-x_{2}\right)-b_{1}\left(\dot{x}_{1}-\dot{x}_{2}\right)\right)
\end{gathered}
$$

The differential equation of motion for unsprung mass $\mathrm{m}_{2}$ is given as in Eq. (39),

$$
\begin{gathered}
m_{2} \ddot{x}_{2}=f_{1}+f_{2}-f_{3}-f_{4} \\
m_{2} \ddot{x}_{2}=k_{1}\left(x_{1}-x_{2}\right)+b_{1}\left(\dot{x}_{1}-\dot{x}_{2}\right)-k_{2}\left(x_{2}-w\right)-b_{2}\left(\dot{x}_{2}-\dot{w}\right)
\end{gathered}
$$

Simplifying the above equations,

$$
\begin{aligned}
& m_{2} \ddot{x}_{2}=k_{1} x_{1}-k_{1} x_{2}+b_{1} \dot{x}_{1}-b_{1} \dot{x}_{2}-k_{2} x_{2}+k_{2} w-b_{2} \dot{x}_{2}+b_{2} \dot{w} \\
& m_{2} \ddot{x}_{2}=-x_{2}\left(k_{1}+k_{2}\right)-\dot{x}_{2}\left(b_{1}+b_{2}\right)+k_{2} w+b_{2} \dot{w}+k_{1} x_{1}+b_{1} \dot{x}_{1}
\end{aligned}
$$




$$
\ddot{x}_{2}=\frac{1}{m_{2}}\left(-x_{2}\left(k_{1}+k_{2}\right)-\dot{x}_{2}\left(b_{1}+b_{2}\right)+k_{2} w+b_{2} \dot{w}+k_{1} x_{1}+b_{1} \dot{x}_{1}\right)
$$

Vertical displacement of the wheel axle for the road excitation input in state space form is given as in Eq. (44) and Eq. (45),

where

$$
\begin{aligned}
& \dot{X}_{q}(t)=A X_{q}(t)+B u_{q}(t) \\
& y_{q}(t)=C X_{q}(t)+D u_{q}(t)
\end{aligned}
$$

$$
\begin{gathered}
\mathrm{X}_{\mathrm{q}}=\left[\begin{array}{ll}
x_{1} & x_{2}
\end{array}\right] \\
u_{q}=w
\end{gathered}
$$

The state matrix are given as follows:

$$
\begin{gathered}
\dot{X}(t)=\left[\begin{array}{cccc}
\frac{-b_{1}}{m_{1}} & \frac{b_{1}}{m_{1}} & \frac{-k_{1}}{m_{1}} & \frac{k_{1}}{m_{1}} \\
\frac{-b_{1}}{m_{2}} & \frac{-b_{1}+b_{2}}{m_{2}} & \frac{k_{1}}{m_{2}} & \frac{-k_{1}+k_{2}}{m_{2}} \\
1 & 0 & 0 & 0 \\
0 & 1 & 0 & 0
\end{array}\right] X(t)+\left[\begin{array}{cc}
0 & 0 \\
\frac{b_{2}}{m_{2}} & \frac{k_{2}}{m_{2}} \\
0 & 0 \\
0 & 0
\end{array}\right]\left[\begin{array}{c}
\dot{w} \\
w
\end{array}\right] \\
Y(t)=\left[\begin{array}{cccc}
0 & 0 & 1 & 0 \\
0 & 0 & 0 & 1 \\
0 & 0 & 1 & -1
\end{array}\right] X(t)+\left[\begin{array}{ll}
0 & 0 \\
0 & 0 \\
0 & 0
\end{array}\right]\left[\begin{array}{c}
\dot{w} \\
w
\end{array}\right]
\end{gathered}
$$

This model is used for analyzing the effect of different road inputs on the vertical displacement of the wheel.

\section{Proposed Method for Estimation of Feedback Motor Current Using Neural Network}

Based on the principle of superposition, the vehicle dynamic parameters such as front wheel angle, vertical displacement and self-aligning moment are combined through scaling and addition for estimating the current to be supplied to the feedback motor as given by Eq. (48),

$$
I_{E}=a\left(X_{2}\right)+b\left(\delta_{f w}\right)+c\left(\tau_{e}\right)
$$

In the above equation, $\mathrm{X}_{2}$ is vertical displacement, $\delta_{\mathrm{fw}}$ is front wheel angle and $\tau_{\mathrm{e}}$ is self-aligning torque. Here the constants $\mathrm{a}, \mathrm{b}$ and $\mathrm{c}$ are considered as the scaling parameters for the vehicle dynamic parameters such as vertical displacement, front wheel angle and self-aligning moment respectively. The magnitude of scale factors can be fixed as per the requirements of producing variable road feel for the driver and it can be modified for the given road conditions. The typical values of scale factors used for simulating the road feel to the driver is shown in Table 1.

Table 1. Scale factors for simulating the road feel.

\begin{tabular}{lccc}
\hline Surface & a & b & c \\
\hline Dry & 5 & 2 & 10 \\
Wet & 3.5 & 4 & 7 \\
Icy & 2.5 & 5.5 & 5 \\
\hline
\end{tabular}


The Eq. (48) provides a fixed magnitude of current to the feedback motor for the given scale factors and vehicle dynamic parameters such as vertical displacement, front wheel angle, self-aligning moment.

In the present work, a back propagation neural network is developed to provide the variable road feel to the driver for the changing road conditions and dynamic states of the vehicle. Proposed neural network uses the vertical displacement; front wheel angle and the aligning moment as inputs and provide the output as the current to be supplied to the feedback motor for generating the torque in the steering wheel as shown in Fig. 6.

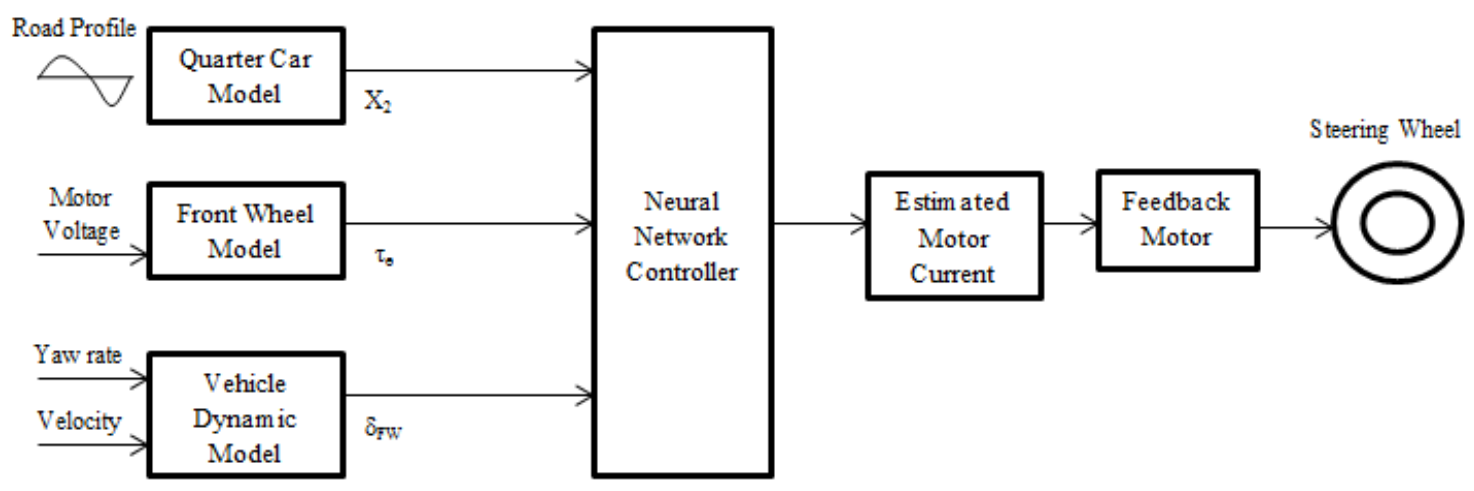

Fig. 6. Proposed neural network based method for feedback torque estimation.

Feedback torque generated in the steering wheel for the given input current can be calculated using the following formula which is given in Eq. (49),

$$
T_{f b}=K_{w m} I_{E}
$$

where $\mathrm{K}_{\mathrm{wm}}$ is the motor constant and $\mathrm{T}_{\mathrm{fb}}$ is the feedback torque.

The target values for the motor current for training the neural network is obtained using the Eq. (48) for the given vertical displacement $\left(\mathrm{X}_{2}\right)$, front wheel angle $\left(\delta_{\mathrm{fw}}\right)$, and self-aligning moment $\left(\tau_{\mathrm{e}}\right)$.

\subsection{Architecture of Proposed Neural Network}

In the present work, three input and single-output feed forward back propagation neural network is used for estimating the current to be supplied to the feedback motor for producing the variable road feel to the driver. The architecture of the neural network is shown in Fig. 7 and it has three input parameters such as vertical displacement $\left(\mathrm{X}_{2}\right)$, front wheel angle $\left(\delta_{\mathrm{fw}}\right)$, and self-aligning moment $\left(\tau_{\mathrm{e}}\right)$.

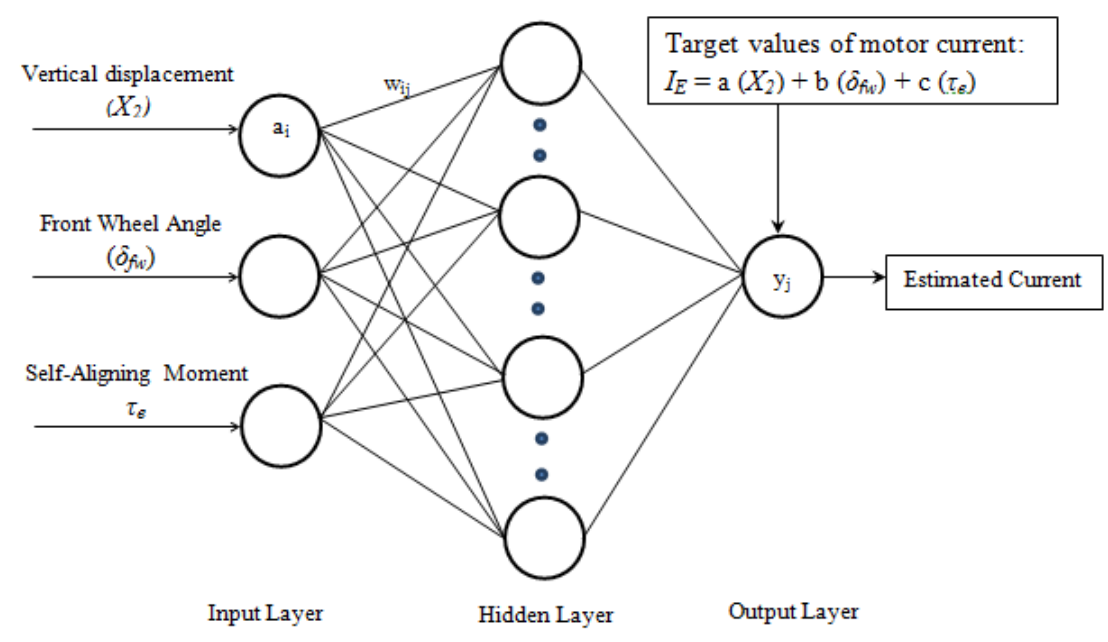

Fig. 7. Schematic description of the proposed back propagation neural network. 
A set of random connection weights are assumed for initiating the training for the proposed neural network. In input layer, a neuron acts as a buffer to distribute the input signals to the hidden layer. This is given as in Eq. (50),

$$
y_{j}=f\left(\sum_{i=1}^{n} w_{j i} a_{i}\right)
$$

The network automatically adjusts its weights based on changes in the input-output pair. The adjustment of the weights is done by the back propagation of the error during training as given in Eq. (51),

$$
\Delta w_{i j}=\eta \delta_{j} a_{i}
$$

where $\eta$ is learning rate and $\delta_{j}$ is a factor which changes according to the input neuron or hidden neuron.

The network includes the input and target values in the training data set and tries to reduce the difference between the target and output values. The neural network learning index helps to minimize the errors between the actual output and desired output which is given in Eq. (52),

$$
J(t)=\frac{1}{2}(e(t))^{2}
$$

where $\mathrm{e}(\mathrm{t})$ is known as an error.

Back propagation algorithm starts with the guess value which could have an impact on squared error value. In order to avoid a large error, neural network is trained for several times. A uniform MSE would ensure that the network is performing well for the unseen data. The sample values of input parameters of the neural network and target values of the motor current for training the proposed neural network is shown in Table 2 for different road profiles.

Table 2. Target values for different road surfaces.

\begin{tabular}{llllll}
\hline \multicolumn{2}{l}{ Sample values of the input parameters of the neural network } & \multicolumn{3}{l}{ Target Points } \\
\cline { 1 - 3 } $\begin{array}{l}\text { Vertical } \\
\text { Displacement(cm) }\end{array}$ & $\begin{array}{l}\text { Front Wheel } \\
\text { Angle(rad) }\end{array}$ & $\begin{array}{l}\text { Self-Aligning } \\
\text { Moment(N-m) }\end{array}$ & Dry & Wet & Icy \\
\hline 1.363343 & 0.010942 & 0.033585 & 1.240002 & 0.844863 & 0.719908 \\
2.532708 & 0.055673 & 0.155245 & 2.113576 & 1.64645 & 1.4988 \\
3.553344 & 0.133148 & 0.351294 & 2.644186 & 2.423075 & 2.352522 \\
4.726248 & 0.237658 & 0.610973 & 3.41954 & 3.345484 & 3.322173 \\
5.92361 & 0.362967 & 0.926046 & 4.364983 & 4.328295 & 4.316742 \\
6.780761 & 0.501766 & 1.283521 & 4.966396 & 5.140485 & 5.195274 \\
7.348215 & 0.645984 & 1.665985 & 5.488022 & 5.797076 & 5.894878 \\
7.582341 & 0.787988 & 2.054928 & 5.94675 & 6.256197 & 5.353909 \\
7.347418 & 0.92047 & 2.431081 & 6.120013 & 6.420451 & 5.515365 \\
6.706671 & 1.036038 & 2.773264 & 6.035915 & 6.310636 & 6.397475 \\
5.387675 & 1.128629 & 3.063515 & 5.047836 & 5.74885 & 5.971364 \\
4.210251 & 1.192937 & 3.285367 & 4.541124 & 5.214002 & 5.427245 \\
3.051814 & 1.225783 & 3.427793 & 4.127231 & 4.624005 & 4.779043 \\
1.782725 & 1.224509 & 3.480294 & 3.475469 & 3.893166 & 3.025318 \\
0.465765 & 1.187838 & 3.436794 & 2.620154 & 3.054748 & 2.191956 \\
-0.00335 & 1.115683 & 3.294364 & 3.254588 & 2.64446 & 1.450896 \\
-1.11132 & 1.010121 & 3.056544 & 2.573227 & 1.773501 & 1.520012 \\
\hline
\end{tabular}

\subsection{Network Parameters}

In the present work, neural network with tangent sigmoid transfer function is selected with a gradient descent learning function. The general parameters used for training the neural network are given in Table 3 . 
Table 3. Properties of the proposed neural network.

\begin{tabular}{lc}
\hline Parameter & Type \\
\hline Training Function & Levenberg-Marquardt back propagation function \\
Adapting Learning Function & Gradient decent with momentum weight and bias learning function \\
Performance Function & Mean squared error \\
Number of Layers & 2 \\
Transfer Function & Tangent sigmoid function \\
No of Hidden Layers & 10 \\
Learning Rate & $100 \%$ \\
Sampling Time & 0.001 \\
\hline
\end{tabular}

\section{Simulation Results and Discussion}

To validate the performance of the neural network method, a simulation study has been carried out for the sinusoidal steering angle driver input and road input. A simulation environment was constructed using the MATLAB / SIMULINK software for the implementation of the proposed method. Vertical displacement of the vehicle, front wheel angle and self-aligning moment of vehicle for the given sinusoidal steering angle input is simulated using the vehicle dynamic models derived in Section 2. The vehicle model parameters considered for the simulation is given in Table 4 .

Table 4. Vehicle parameter values.

\begin{tabular}{lcc}
\hline Symbol & Magnitude & Unit \\
\hline $\mathrm{G}_{\mathrm{mr}}$ & $7.367 * 10^{-3}$ & $\mathrm{M}$ \\
$\mathrm{M}_{\mathrm{r}}$ & 2.000 & $\mathrm{Kg}$ \\
$\mathrm{C}_{\mathrm{tr}}$ & $6.72^{*} 10^{-5}$ & $\mathrm{rad} / \mathrm{Nm}$ \\
$\mathrm{B}_{\mathrm{tr}}$ & 40.000 & $\mathrm{Nm} \mathrm{s} / \mathrm{rad}$ \\
$\mathrm{J}_{\mathrm{wm}}$ & $9.8^{*} 10^{-4}$ & $\mathrm{Kg} \mathrm{m}{ }^{2}$ \\
$\mathrm{~B}_{\mathrm{wm}}$ & $5.7 * 10^{-4}$ & $\mathrm{~N} / \mathrm{m} / \mathrm{s})$ \\
$\mathrm{C}_{\mathrm{wm}}$ & $1.29^{*} 10^{-3}$ & $\mathrm{Nm} \mathrm{rad} / \mathrm{s}$ \\
$\mathrm{m}_{1}$ & 466.50 & $\mathrm{Kg}$ \\
$\mathrm{m}_{2}$ & 49.80 & $\mathrm{Kg}$ \\
$\mathrm{k}_{1}$ & 5700 & $\mathrm{~N} / \mathrm{m}$ \\
$\mathrm{k}_{2}$ & 15000 & $\mathrm{~N} / \mathrm{m}$ \\
$\mathrm{b}_{1}$ & 290 & $\mathrm{~N} /(\mathrm{m} / \mathrm{s})$ \\
$\mathrm{b}_{2}$ & 1400 & $\mathrm{~N} /(\mathrm{m} / \mathrm{s})$ \\
$\mathrm{G}_{\mathrm{r}}$ & 0.49 & - \\
$\mathrm{K}_{\mathrm{wm}}$ & 2.00 & $\mathrm{Amp} / \mathrm{N}-\mathrm{m}$ \\
$\mathrm{R}_{\mathrm{wa}}$ & 0.21 & $\mathrm{Ohm}$ \\
$\mathrm{L}_{\mathrm{wa}}$ & $0.52^{*} * 10^{-3}$ & $\mathrm{Henry}$ \\
$\mathrm{B}_{\mathrm{r}}$ & 90 & $\mathrm{Nm} \mathrm{s} / \mathrm{rad}$ \\
$\mathrm{B}_{\mathrm{t}}$ & 40 & $\mathrm{Nm} \mathrm{s} / \mathrm{rad}$ \\
$\mathrm{K}_{\mathrm{tr}}$ & 2.00 & $\mathrm{Amp} / \mathrm{N}-\mathrm{m}$ \\
$\mathrm{C}_{\alpha, \mathrm{f}}$ & 165000 & $\mathrm{~N} / \mathrm{rad}$ \\
$\mathrm{C}_{\alpha, \mathrm{r}}$ & 165000 & $\mathrm{~N} / \mathrm{rad}$ \\
$\mathrm{I}_{\mathrm{z}}$ & 1808 & $\mathrm{Kg} \cdot \mathrm{m}^{2}$ \\
$\mathrm{~m}$ & 1300 & $\mathrm{Kg}$ \\
$\mathrm{l}_{\mathrm{f}}$ & 1.22 & $\mathrm{~m}$ \\
$\mathrm{l}_{\mathrm{r}}$ & 1.22 & $\mathrm{~m}$ \\
$\mathrm{~J}_{\mathrm{t}}$ & 1.8 & $\mathrm{Kg} \cdot \mathrm{m}^{2}$ \\
$\mathrm{C}_{\mathrm{f}}$ & 165000 & $\mathrm{~N} / \mathrm{rad}$ \\
$\mathrm{V}$ & 0.035 & $\mathrm{~m} / \mathrm{s}$ \\
$\mathrm{t}_{\mathrm{p}}$ & & - \\
$\mathrm{t}_{\mathrm{m}}$ & & - \\
\hline & & \\
\hline & & \\
\hline
\end{tabular}


Three different road profiles have been considered for the simulation and it is shown in Fig. 8. The parameters used for the road profile simulation using the quarter car model are shown in Table. 5.

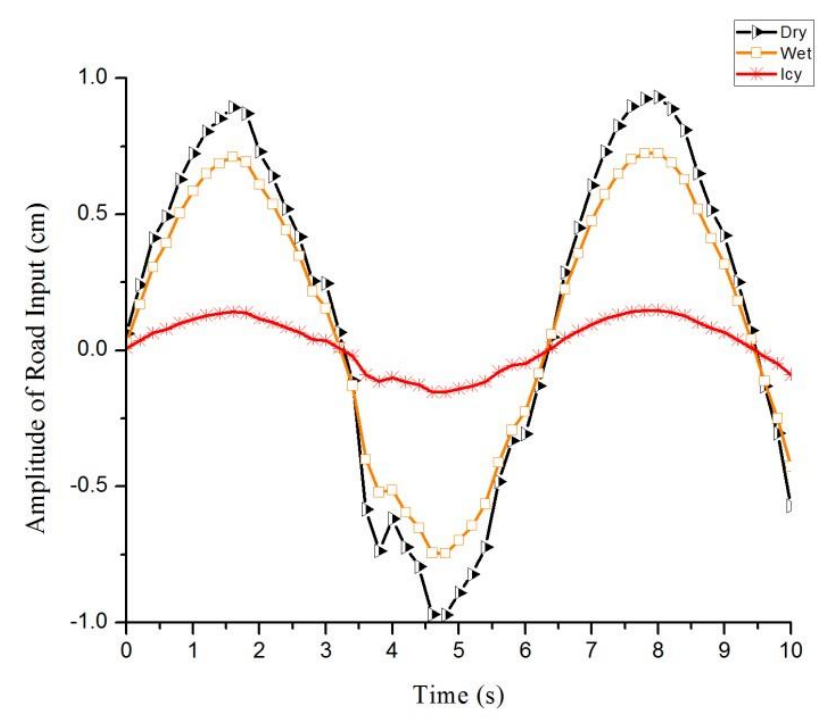

Fig. 8. Simulated road profiles.

Table 5. Model parameter values for simulating sinusoidal road surfaces using quarter car model.

\begin{tabular}{lcc}
\hline Types of surfaces & Vertical stiffness $\left(\mathrm{k}_{2}\right)(\mathrm{N} / \mathrm{m})$ & Amplitude of sine wave road profile $(\mathrm{w})(\mathrm{cm})$ \\
\hline Dry & 15000 & 0.9 \\
Snow & 8000 & 0.5 \\
Icy & 3000 & 0.3 \\
\hline
\end{tabular}

The simulation of vehicle dynamic models and training results of proposed neural method for different road surfaces such as dry, wet and icy are presented and discussed in the subsequent sections.

\subsection{Simulated Vehicle Dynamic Parameters}

The input parameters of the neural network model such as vertical displacement, front wheel angle and self-aligning moment are simulated using the vehicle dynamic models for the sinusoidal steering angle driver input and sinusoidal dry road surface. The steering wheel angle which is given by the driver and the front wheel angle are shown in Fig. 9. 


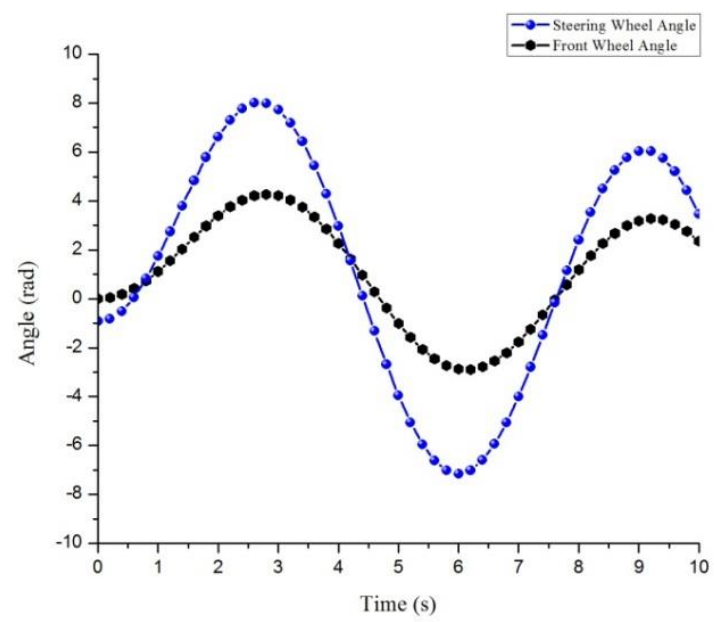

(a) Steering wheel angle and front wheel angle.

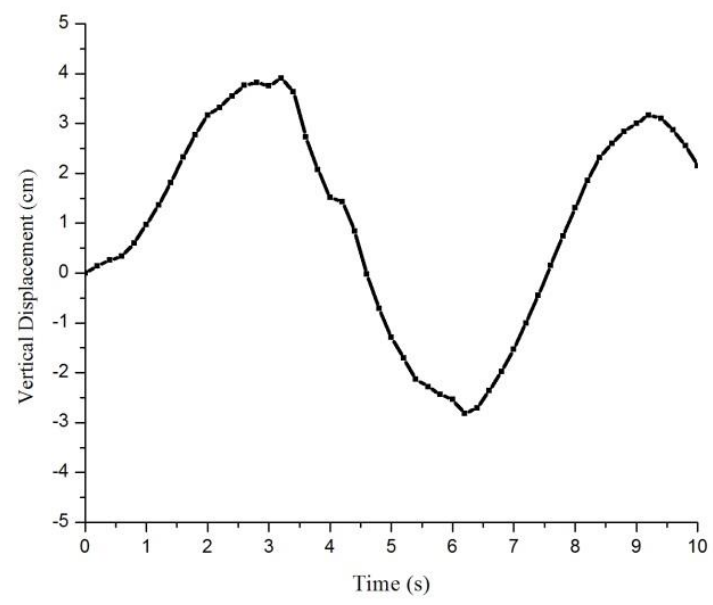

(b) Vertical displacement.

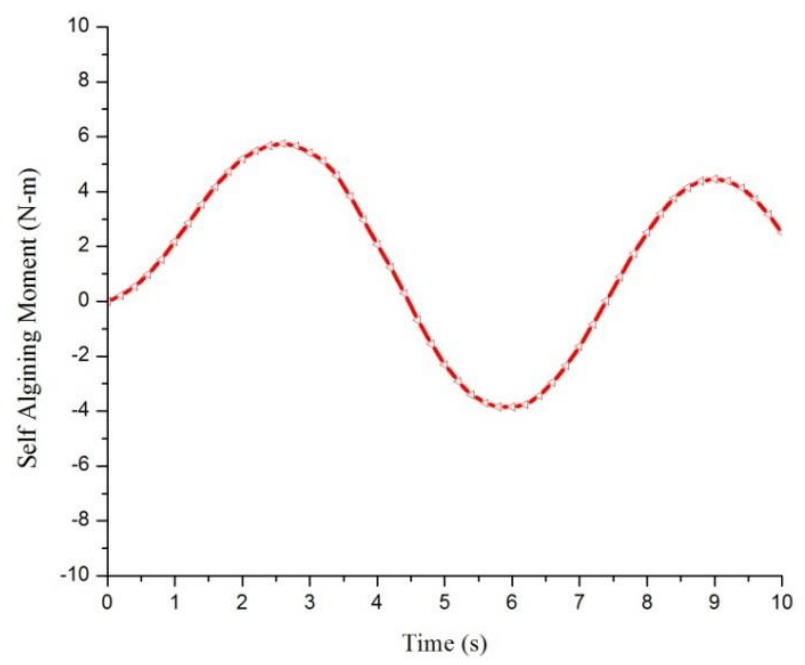

(c) Self-aligning moment.

Fig. 9. Simulated values of vehicle dynamic parameters. 
It can be seen that the front wheel angle follows sinusoidal path due to the sinusoidal driver input which shows the directional stability and maneuvering of the vehicle. It is also noted that vertical displacement follows the shape of the dry road profile input.

\subsection{Estimated Motor Current Using the Proposed Neural Network}

Proposed neural network is trained to produce the current values to be supplied to the feedback motor in the steering wheel for the given vehicle dynamic parameters. Table 6 shows the sample values of the estimated motor current using the neural network for the different road surfaces. It can be noticed that there is a variation in the magnitude of the motor current for the different road surfaces for the given vehicle dynamic parameters.

Table 6. Sample values of input and output parameters of the neural network.

\begin{tabular}{|c|c|c|c|c|c|}
\hline \multicolumn{3}{|c|}{ Sample values of the input parameters of the neural network } & \multirow{2}{*}{\multicolumn{3}{|c|}{$\begin{array}{l}\text { Estimated Current values using the neural } \\
\text { network for different road surfaces(Amps) }\end{array}$}} \\
\hline \multirow{2}{*}{$\begin{array}{l}\text { Vertical } \\
\text { Displacement }(\mathrm{cm})\end{array}$} & \multirow{2}{*}{$\begin{array}{l}\text { Front Wheel } \\
\text { Angle(rad) }\end{array}$} & \multirow{2}{*}{$\begin{array}{l}\text { Self-Aligning } \\
\text { Moment(N-m) }\end{array}$} & & & \\
\hline & & & Dry & Wet & Icy \\
\hline 1.363343 & 0.010942 & 0.033585 & 1.239795 & 0.844722 & 0.719788 \\
\hline 2.532708 & 0.055673 & 0.155245 & 2.113224 & 1.646176 & 1.49855 \\
\hline 3.553344 & 0.133148 & 0.351294 & 2.643745 & 2.422671 & 2.35213 \\
\hline 4.726248 & 0.237658 & 0.610973 & 3.41897 & 3.344927 & 2.321619 \\
\hline 5.92361 & 0.362967 & 0.926046 & 4.364256 & 4.327574 & 3.316023 \\
\hline 6.780761 & 0.501766 & 1.283521 & 4.965569 & 5.139628 & 4.194408 \\
\hline 7.348215 & 0.645984 & 1.665985 & 5.487107 & 5.79611 & 4.893895 \\
\hline 7.582341 & 0.787988 & 2.054928 & 5.945759 & 6.255154 & 5.35285 \\
\hline 7.347418 & 0.92047 & 2.431081 & 6.118993 & 6.419381 & 5.514279 \\
\hline 6.706671 & 1.036038 & 2.773264 & 6.03490 & 6.309584 & 5.396409 \\
\hline 5.387675 & 1.128629 & 3.063515 & 5.046994 & 5.747892 & 4.970369 \\
\hline 4.210251 & 1.192937 & 3.285367 & 4.540367 & 5.213133 & 4.426341 \\
\hline 3.051814 & 1.225783 & 3.427793 & 4.126543 & 4.623234 & 3.778246 \\
\hline 1.782725 & 1.224509 & 3.480294 & 3.47489 & 3.892517 & 3.024648 \\
\hline 0.465765 & 1.187838 & 3.436794 & 2.619718 & 3.054239 & 2.191424 \\
\hline-0.00335 & 1.115683 & 3.294364 & 3.254045 & 2.644019 & 1.450488 \\
\hline-1.11132 & 1.010121 & 3.056544 & 2.572799 & 1.773205 & 1.519758 \\
\hline
\end{tabular}

Figure 10 shows the estimated current using the proposed neural network method and target values supplied to the network using vehicle dynamic model for the dry road profile.

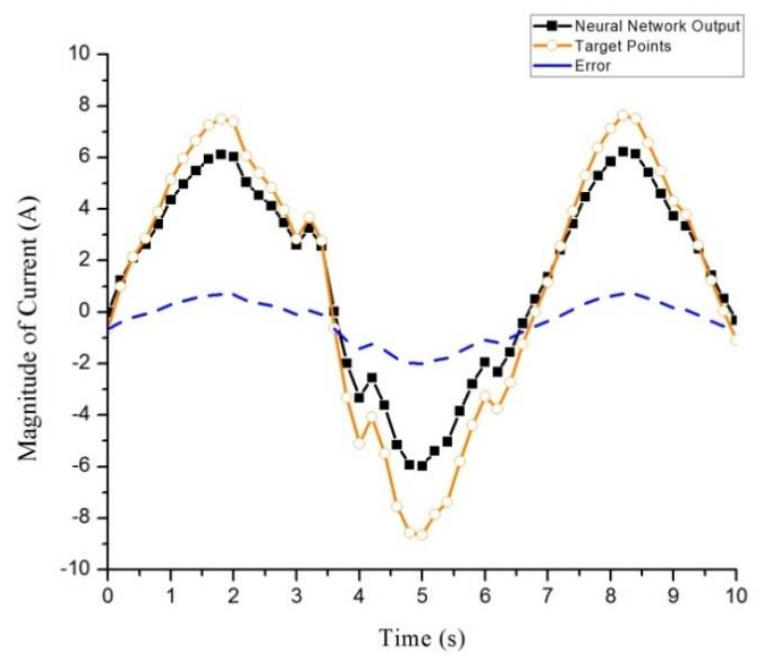

Fig. 10. Training and validation results of neural network model for the dry road surface. 
It can be observed that the proposed neural method is able to produce the motor current with a smaller difference in the magnitude of 1 Amps at the peak points, and it follows the general trend of the given dry road profile and the target values of motor current. The mean squared error of between the target value and output of the neural network is found to be in the order of $3 \times 10^{-3}$ Amps.

\subsection{Estimated Feedback Torque for Road Profiles}

The estimated current of the proposed method is supplied to the feedback motor in the steering wheel for producing the variable feedback torque. Based on the motor constant value, the feedback torque produced by the motor was calculated for the various road profiles and it is shown in Fig. 11.

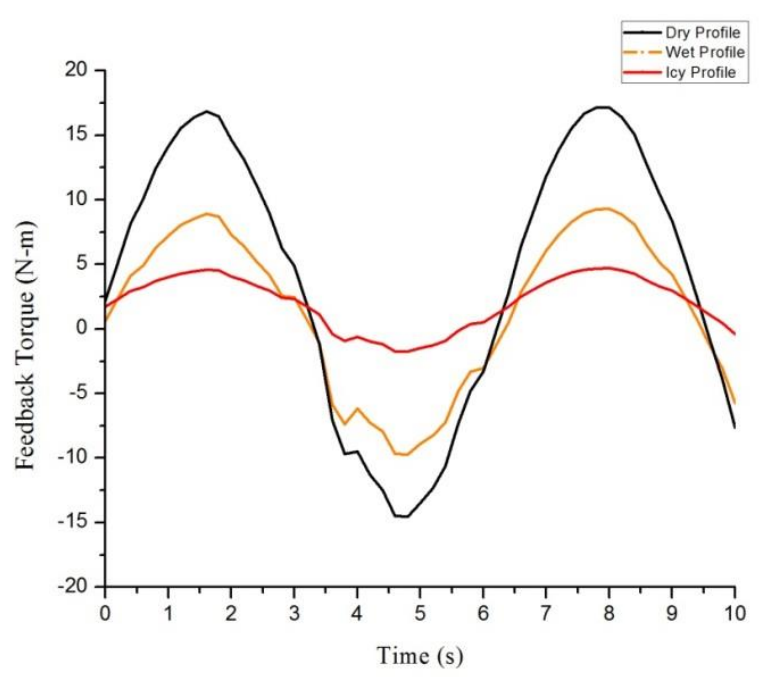

Fig. 11. Effect of road surface on feedback torque in the steering wheel.

From the graph it is clear that the varying magnitude of feedback torque is produced for the different road conditions. From the above simulation results, it is proved that the proposed neural network method provides a flexibility of producing the varying feedback torque based on the different road inputs.

\section{Conclusions}

Generating the road feel is an important criterion in steer-by-wire vehicle systems. This paper proposed a neural network based methodology for generating the road feel to the driver based on the vehicle dynamic parameters such as, vertical displacement, front wheel angle and self aligning moment. Based on the principle of superpoistion, a multi linear model of the vehicle dynamic parameters is assumed for generating the motor current values to be supplied to the feedback motor in the steering wheel and it is furhter used for training the neural network to produce the variable road feel to the driver. Performance of the proposed neural network method was tested for different sinusoidal road profiles and sinusoidal steering angle driver input. Simulation results confirm that the estimated current using the neural network follows the general trend of the input road profile with a mean squared error of $3 \times 10^{-3}$ Amps. Also it was noted that its magnitude varies for the diffrent road surfaces significantly. It proves that the proposed method has an advantage of producing the road feel to the driver for the changing the road profiles adaptively. The proposed method used simple measurable quantities such as front wheel angle and vertical displacement of the front axle for generating the road feel, hene it can be implemented with the suitable instrumentations in the steer by wire vehciles.

\section{References}

[1] Y. Shengbing, D. Chunan, J. Xuewu, and C. Kuiyuan, "Research on road feeling control strategy of steer-by-wire," SAE Tech Pap, 2007-01-3652, 2007. 
[2] S. E. Mohamed and S. A. Albatlan, "Modelling and experimental design approach for integration of conventional power steering and a steer-by-wire system based on active steering angle control," Am J Vebicle Des, vol. 2, no. 1, pp. 32-42, 2014.

[3] B. H. Wang, X. B. Chen, and H. Y. Zhao, "Application study of active front steering with integrated feedback control for steer-by-wire system," Int J Mech Syst Eng, vol.3, pp.148-153, Nov. 2013.

[4] H. Zheng, C. Zong, and L. Yu," Road feel feedback design for vehicle steer-by-wire via electric power steering," SAE Technical Paper, 2013-01-2898, 2013.

[5] R. Kazemi and I. Mousavinejad, "A Comprehensive model for developing of steer-by-wire system," Int J Mech Eng, vol.5, no.8, 2011.

[6] B. Zheng and S. Anwar, "Fault-tolerant control of the road wheel subsystem in a steer-by-wire system," Int J Vehicular Tech, 2008.

[7] I. Mousavinejad and R. Kazemi," Variable structure controller design for steer-by-wire system of a passenger car," J Mech Sci Tech, vol. 28, no. 8, pp. 3285-3299, 2014.

[8] Y. Han, L. He, X. Wang, and C. Zong," Research on torque ratio based on the steering wheel torque characteristic for steer-by-wire system," J Applied Mathematics, vol. 2014, pp. 1-7, 2014.

[9] H. Na, C. Zong, and D. Hu, "Investigations on cornering control algorithm design and road feeling optimization for a steer-by-wire vehicle," in International Conference on Mechatronics and Automation, Changchun, China, 2009, pp. 3246-3251.

[10] A. Marouf, M. Djemaï, C. Sentouh, and P. Pudlo, "Driver torque and road reaction force estimation of an electric power assisted steering using sliding mode observer with unknown inputs," in 13th International IEEE Annual Conference on Intelligent Transportation Systems, Portugal, 2010, pp. 354-359.

[11] S. M. H. Fahami, H. Zamzuri, and S. Amrimazlan, "Development of estimation force feedback torque control algorithm for driver steering feel in vehicle steer by wire system: hardware in the loop," Int $J$ Vebicular Tech, pp. 1-17, 2015.

[12] C. Peng, P. A. Cowel, C. J. Chisholm, and J. A. Lines, "Lateral tyre dynamic characteristics," J Terramechanics, vol. 31, pp. 395-414, 1994.

[13] A. Florin, M.R.I Cozmin, and P. Liliana, "Passive suspension modelling using MATLAB, quarter car model, input signal step type," TEHNOMUS, pp. 258-263, 2013.

[14] N. Bajcinca, C. Nuthong and F. Svaricek, "Road feedback estimation for steer-by-wire control," in International Conference on Control Applications, Munich, 2006, pp. 1288-1293.

[15] P. Guarneri, G. Rocca, and M. Gobbi, "A neural network based model for the dynamic simulation of the tire/suspension system while traversing road irregularities," IEEE T Neural Networks, vol. 19, pp. 1549-1563, 2008.

[16] I. Eski and A. Temurlenk, "Design of neural network based control systems for active steering system," Non-Linear Dynam, vol. 73, no. 3, pp. 1443-1454, 2013.

[17] J. Iqbal, K. Shin, and C. S. Han, "Neural network based control for steer-by-wire systems vehicles," Adv Circuits Syst Signal Process Telecommun, pp. 69-74, 2014.

[18] N. Lin, C. Zong, M. Tomizuka, P. Song, Z. Zhang, and G. Li, “An overview on study of identification of driver behaviour characteristics for automotive control," Math Probl Eng, pp. 1-15, 2014.

[19] M. Yanan, F. Xiuwei, and F. Li, "Application of neural network trained by adaptive particle swarm optimization to fault diagnosis for steer-by-wire system," in International Conference on Measuring Technology and Mechatronics Automation, 2010, pp.652-655.

[20] J. H. Sohn, S. K. Lee, and W. S. Yoo, "Hybrid neural network bushing model for vehicle dynamics simulation," J Mech Sci Tech, vol. 22, no. 2, pp. 2365-2374, 2008.

[21] Y. Lin, P. Tang, W. J. Zhang, and Q. Yu, "Artificial neural network modelling of driver handling behaviour in a driver-vehicle-environment system," Int J Vehicle Des, vol. 37, no. 1, pp. 24-45, 2005.

[22] J. Judycki and P. Jaskula, "Structural design and sensitivity analysis of semi-rigid pavement of a motorway,” Engineering Journal, vol. 16, no. 4, pp. 117-126, 2012.

[23] P. Yih, J. Ryu, and J. C. Gerdes, "Vehicle state estimation using steering torque," Proceeding of the 2004 American Control Conference, 2004, pp. 2116-2121. 